\title{
BEND PROPAGATION BY A SLIDING FILAMENT MODEL FOR FLAGELLA
}

\author{
BY C. J. BROKAW* \\ Department of Zoology, University of Cambridge
}

(Received 5 March 1971)

\section{INTRODUCTION}

The important electron-microscope studies by Satir $(1965,1968)$ have demonstrated that the bending of a cilium is accompanied by longitudinal sliding of its peripheral microtubular filaments relative to one another. These observations imply that the bending of the cilium is not generated by active contraction or extension of the filaments, since they appear to retain constant lengths throughout the bending cycle. More probably, bending is generated by an active sliding process involving filament interactions analogous to those occurring in the active sliding process which is believed to be the basis for muscular contraction. Support for Satir's conclusions can also be drawn from the manner in which filaments are interconnected in an unusual compound cilium (Horridge, 1965) and from some observations on the form of the movement of spermatozoa in the presence of an inhibitor, thiourea (Brokaw, I965).

Satir (1967, 1968) and others (Sleigh, 1968; Brokaw, 1968) have discussed some aspects of sliding-filament models for ciliary and flagellar movement. On the other hand, most attempts to deal quantitatively with the mechanics of wave propagation by flagella have been based on a 'local bending' type of model, in which the active moment and the bending of the flagellum at a particular point are considered to be generated by active processes at that point within the flagellum (Machin, 1958; Brokaw, 1966a). These discussions of wave propagation have attempted to explain the ability of flagella to propagate bending waves by making simple assumptions about the local characteristics of the active process, so that no higher-level system for co-ordinating bending is required. These proposals for wave propagation have encountered some serious difficulties, which have been discussed in previous papers (Brokaw, 1962, 1968, 1970).

In this paper I have attempted to define some of the quantitative properties of a sliding filament model for flagellar movement, with particular reference to the generation of the bending moments required to balance the viscous moments imposed on the flagellum by its movement in a viscous medium. It will be shown that the difficulties which have hindered previous attempts to understand the control of bend propagation in flagella can be resolved by consideration of a sliding filament model, and that this model suggests how a relatively simple mechanism for the control of the active process can explain the generation of propagated bending waves by flagella.

\footnotetext{
* Permanent address, to which reprint requests should be sent: Division of Biology, California Institute of Technology, Pasadena, California 9r Io9.
} 
SPECIFICATION OF THE PROPERTIES OF A SLIDING-FILAMENT MODEL

For the purposes of this paper a flagellum can be represented by a system consisting of two parallel filaments. An $x, y$ co-ordinate system is defined in the plane of these filaments. Only bending of the flagellum in this $x, y$ plane will be considered. This paper will be primarily concerned with uniform, symmetrical bending waves which have the $x$ axis as their axis of symmetry. The base of the flagellum will be near $x=0$, and the distal end of the flagellum will be located in the positive direction along $x$. Distance along the length of the flagellum will be measured by the co-ordinate, $s$, with $s=0$ at the base of the flagellum and $s=S$ at the distal end of the flagellum. For small-amplitude bending waves, $S$ will also represent the value of $x$ at the distal end of the flagellum.

(a)

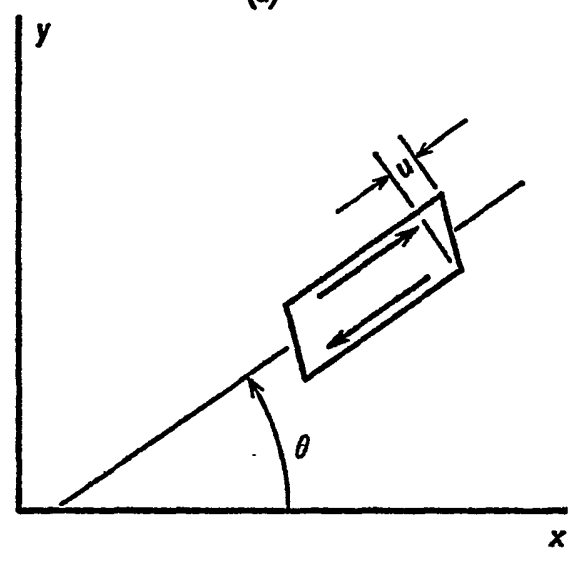

(b)

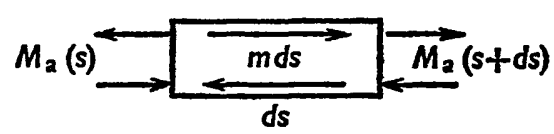

(c)

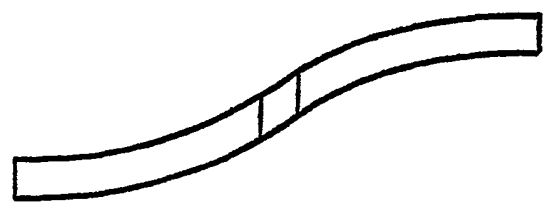

Fig. I. (a) An element along the length of the flagellar model, with arrows to indicate the direction of positive shear. (b) The balance of active moment, $M_{a}$, on an element along the length of the flagellar model, in which an active shear moment, $m d s$, is generated. (c) Flagellar bending caused by positive active shear in a small length of the flagellum.

The curvature of the flagellum at any point is defined in the usual manner as $d \theta / d s$, where $\theta$ is an angle between a tangent to the flagellum at $s$ and the positive $x$ axis, as illustrated in Fig. I $(a)$. The curvature will be designated by $\kappa . \kappa=\mathrm{I} / \rho$, where $\rho$ is the radius of curvature, as in previous papers (Brokaw, $1965,1966 a$ ).

The flagellar filaments will be considered to be inextensible, so that all bending of the flagellum must be accommodated by longitudinal shear between the filaments. Let the magnitude of the longitudinal shear displacement between the filaments at any point $s$ along their length be represented by $u$. When the flagellum is at rest in a completely straight configuration $u$ will be assumed to be zero throughout the flagellum. When the flagellum is bent, the difference between the val res of $u$ at any two points along the flagellum will be proportional to the angle between tangents to the flagellum at these two points, and in the limit,

$$
d u / d s=r d \theta / d s=r \kappa .
$$

The spacing between the filaments is represented by $r$ in equation ( $I$ ); it is assumed to remain contant along $s$. Equation (I) defines the direction of positive shear as that indicated in Fig. $I(a)$. 
An active process which tends to cause longitudinal shear is assumed to occur between the filaments. This active process will apply longitudinal shearing forces to the filaments, which will be measured by a moment, $m$, per unit length of flagellum. Forces applied in the direction which will cause positive shear, $u$, will be considered to generate positive values of $m$. These locally generated forces or moments will add up along the length of the flagellum to give the total active moment at any point. If this total active moment is represented by $M_{a}$, then in each element, $d s$, along the length of the flagellum,

$$
M_{a}(s)=M_{a}(s+d s)+m d s,
$$

as illustrated in Fig. $\mathbf{I}(b)$. Consequently,

$$
d M_{a} / d s=-m .
$$

$M_{a}$ is considered to be positive when applied as shown in Fig. $I(b)$. It is therefore equivalent to the case where a positive $M_{a}$ generated within an element, $d s$, by a local bending mechanism will produce a positive curvature in the element, $d s$.

If the flagellum contains some resistance to longitudinal shear between the filaments, the shear generated by the active process will tend to bend the flagellum. The effect of positive shear in an element, $d s$, will be to produce positive curvature $(+\kappa)$ to the left of $d s$ (towards $s=0$ and the base of the flagellum) and negative curvature $(-\kappa)$ to the right of $d s$, as illustrated in Fig. I $(c)$. The shape of the flagellum at any time will be determined by the distributions of the active moment generated within the flagellum, the external moment applied to the flagellum by viscous forces if the flagellum is moving through a viscous medium, and the internal moments resulting from elastic resistances to bending of the filaments and to longitudinal shear between the filaments.

The moment applied to the flagellum by the external viscous forces is represented by $M_{v}$. For a nearly straight flagellum, $M_{v}$ can be obtained by integration of

$$
d^{2} M_{v} / d s^{2}=-C_{N} V_{N}
$$

a more complicated function is required for flagella with large-amplitude curves. This problem is discussed in greater detail elsewhere (Brokaw, 1970). In equation (4), $C_{N}$ is the viscous drag coefficient for movement normal to the flagellum; this quantity is represented by $\beta$ in the paper by Machin (1958). $V_{N}$ is the component in the direction normal to the flagellum of the velocity of the flagellum relative to the viscous medium. This approach results in the definition of positive $M_{v}$ as a moment which tends to cause positive curvature of the flagellum. Consequently, the equation for balance between active and viscous moments at any point will be

$$
M_{a}+M_{v}=\text { o, }
$$

and, from equation (3), this also implies that

$$
-m+d M_{v} / d s=0 .
$$

If the flagellum contains significant internal elastic resistances, equation (5) must be expanded to contain an elastic bending moment, $M_{e}$ :

$$
M_{a}+M_{v}+M_{e}=\text { o. }
$$

For a homogeneous flagellum, with simple elastic properties, $M_{e}$ at any point will be proportional to the curvature at that point. This can be described by $M_{e}=-\alpha \kappa$, 
where $\alpha$ is an elastic constant for the bending resistance of the flagellum. Machin (I958) used $\alpha$ in a similar manner, except for the sign convention. In the present paper, a positive $M_{e}$ is one which tends to cause positive curvature.

If a significant amount of shear can occur within the flagellum, as in the model outlined here, its elastic resistance to bending cannot be represented by a single constant, $\alpha$. The elastic moment at a particular point will not be a simple function of the curvature at that point, but will depend also on the distribution of shear throughout the flagellum and the boundary conditions for shear at the ends of the flagellum. With the simple model described here, $M_{e}$ will have two components. The first component will be the result of the bending resistance of the individual filaments, represented by $\alpha_{0}$, as in Brokaw (I966a). This will contribute to $M_{e}$ a component equal to $-2 \alpha_{0} \kappa$. The second component will result from the longitudinal shear resistance per unit length, represented by $\alpha_{2}$, as in Brokaw (I966a). A positive shear, $u$, will be opposed by elastic resisting forces equal to $-\alpha_{2} u$. As in equation (3), the elastic moment, $M_{e}$, resulting from this shear displacement will be given by

Using equation (I),

$$
d M_{e} / d s=r \alpha_{2} u \text {. }
$$

$$
d^{2} M_{e} / d s^{2}=r \alpha_{2} d u / d s=r^{2} \alpha_{2} \kappa .
$$

If equation (7) is differentiated twice with respect to $s$, the component of the elastic moment resulting from the shear resistance (equation (9)) can be combined with the other moments to give

$$
-d m / d s+d^{2} M_{v} / d s^{2}-2 \alpha_{0} d^{2} \kappa / d s^{2}+r^{2} \alpha_{2} \kappa=0 .
$$

Equation (Io) can also be obtained from the relationships discussed in Brokaw (1966a) and in Lubliner \& Blum (1971).

Bending waves can be propagated along a passive elastic filament in a viscous medium if one end of the filament is caused to oscillate by an active process. If the amplitude of the oscillation is small, the length of the waves will be determined by the elastic constants of the flagellum, the viscosity of the medium, and the frequency of the driving oscillator. However, Machin (1958) showed that the amplitude of such wave movement would diminish noticeably over lengths of the order of one wavelength. Since flagella clearly can propagate waves of maintained amplitude for several wavelengths (e.g. Gray, r955; Brokaw \& Wright, r963), active bending moment must be generated throughout the flagellum. The ability of the flagellum to generate the appropriate amount of active moment to satisfy equations (7) or (10) throughout the flagellum must be explained by any adequate theory for the control of bend propagation by flagella.

The possibility that a relatively simple controlling relationship between the curvature of the flagellum and the magnitude of the active process might provide the proper balance between active and resisting moments, and explain the co-orciination required for flagellar wave propagation, was first discussed in detail by Machin (1958, I963). He considered the case of a local bending model in which $M_{a}$ was controlled by a function of $\kappa$. In such a flagellum, wave propagation can occur automatically, if a time lag is introduced into the relationship between $M_{a}$ and $\kappa$. Some of the difficulties with this model have been mentioned in previous papers (Brokaw, I962, I966 $a, b$, I968). With a local bending model, wave propagation could also result if the rate of bending, 
$d \kappa / d t$, were controlled by a function of $\kappa$, again with a time delay; this mechanism is subject to some of the same difficulties. A different type of control mechanism for bend propagation, in which bending is activated by the local curvature induced by shear just ahead of a region in which $d \kappa / d t$ is concentrated, was discussed by Brokaw (1966a) and by Lubliner \& Blum (1971). This mechanism, which was also developed in terms of a local bending model, assumes that the shear resistance of the flagellum is relatively high.

With a sliding-filament model, bending-wave propagation can also occur automatically if the active process is controlled by $\kappa$. If negative $\kappa$ in a bent region activates positive shear, the active process will tend to cause negative $\kappa$ to the right of the bent region and positive $\kappa$ to the left of the bent region, as in Fig. I $(c)$. This is equivalent to the propagation of the bend from left to right along the flagellum, and is closely related to the mechanism for bend propagation by shear proposed earlier in terms of a local bending model (Brokaw, I966a). Either $m$ or $d u / d t$ might be controlled by $\kappa$. Consideration of the distribution of $d u / d t$ in a flagellum when it is bending in the manner described by Brokaw (1970) does not suggest any simple relationship for the control of $d u / d t$ by $\kappa$. In this paper the possibility that $m$ is controlled by $\kappa$ will be examined in detail. This control situation is, of course, closely related to Machin's original proposal for the control of $M_{a}$ by $\kappa$.

In discussing these control mechanisms for the active process in flagella, it will be convenient to use the transfer-function terminology applied to this problem by Machin (1963). A transfer function is a convenient mathematical formalism for dealing with situations where the output of a system depends not only on the present value of the input, but also on values of the input and the output at previous times. A useful discussion of the application of this concept to the analysis of biological control systems has been presented by Machin (1964). For flagella, the problem becomes one of finding a transfer function for the control of $m$ by $\kappa$, which when included in equation (10) will yield solutions which match the observed movement of flagella. This problem can be approached in two ways: a description of the movement can be combined with equation (10), in order to obtain information about the nature of the transfer function required to produce that movement; or, a particular transfer function can be combined with equation (IO), and a solution for the resulting movement obtained, for comparison with observations. In the present paper the first of these approaches will be used to obtain some general information about the transfer function required for bend propagation by flagella.

Machin (1958) and Rikmenspoel $(1965,1966)$ have argued that the bending waves on an active flagellum should have the same wavelength as the waves generated by oscillation of one end of a passive elastic filament at similar values of frequency and viscosity. Values for the bending resistance, $\alpha$, have then been estimated from the parameters of flagellar wave movement by using the equation which relates $\alpha$ to the viscosity, wavelength, and frequency of small-amplitude oscillation of a passive elastic filament. These values for the bending resistance correspond to the situation where $M_{e}$ and $M_{v}$ have similar magnitudes. This argument has never been supported by proof, and is quite unnecessary. Unfortunately, there are no independent measurements of the elastic constants of flagella.

The biological usefulness of cilia and flagella depends on their ability to work 
against the viscous resistances presented by the medium in which they are operating. It might be expected that, for efficient movement, other resistances should be kept reasonably small; in any event, the balance between $M_{a}$ and $M_{v}$ is the major biological problem. In the next section of this paper the balance between $M_{a}$ and $M_{v}$ will be considered for the limiting case where $M_{e}$ is negligible. The effects of elastic resistance will be introduced in a subsequent section, but will not be considered in detail.

\section{THE BALANCE BETWEEN ACTIVE AND VISCOUS BENDING MOMENTS}

Small-amplitude sinusoidal waves on a flagellum of infinite length

The mathematically simple case of small-amplitude sinusoidal movement provides a useful introduction to the problem of the control of undulatory flagellar movement, even though the amplitudes of flagellar bending waves are seldom small enough to justify direct numerical application of the results. For small-amplitude wave movement, $s$ and $x$ are considered to be equivalent, and conditions at the ends of the flagellum are usually ignored by assuming that the ends of the flagellum are at - and + infinity.

Small-amplitude sinusoidal bending waves on a flagellum may be described by the functions

$$
y=b \cos 2 \pi(f t-x / \lambda) \text { or } y=b e^{i 2 \pi(f t-x / \lambda)} .
$$

In the usual manner, $b, f$ and $\lambda$ measure, respectively, the amplitude, frequency and wavelength of the waves on the flagellum. The viscous bending moment imposed on the flagellum as a result of this movement is then given by

$$
\begin{aligned}
d^{2} M_{v} / d x^{2} & =-C_{N} d y / d t \\
& =+C_{N} 2 \pi f b \sin 2 \pi(f t-x / \lambda),
\end{aligned}
$$

from equation (4). Integration of equation (12) leads to

and

$$
d M_{v} / d x=C_{N} 2 \pi f b(\lambda / 2 \pi) \cos 2 \pi(f t-x / \lambda)
$$

$$
M_{v}=-C_{N} 2 \pi f b(\lambda / 2 \pi)^{2} \sin 2 \pi(f t-x / \lambda) .
$$

For small-amplitude sinusoidal movement the curvature of the flagellum is given by

$$
\kappa=d^{2} y / d x^{2}=-b(2 \pi / \lambda)^{2} \cos 2 \pi(f t-x / \lambda) .
$$

Comparison of equations ( $\left.1_{4}\right)$ and (I5) indicates that $M_{v}$ and $\kappa$ differ in phase by $\frac{1}{4}$ cycle. Therefore, for a local bending model in which $M_{a}$ is controlled so that equation (5) is satisfied, a transfer function for the control of $M_{a}$ by $\kappa$ must introduce a phase lag of $\frac{1}{4}$ cycle between $M_{a}$ and $-\kappa$. This phase lag must be independent of the frequency. Since flagella operate over a wide range of frequencies, a frequencyindependent phase lag cannot be generated in a simple manner by a constant time delay or an exponential time delay between the curvature and the active moment. The absence of any reasonable biological mechanism for introducing this frequencyindependent phase lag has been one of the principal objections to Machin's original proposal that flagellar movement is controlled by a simple relationship between the intensity of the active process at a particular point and the curvature at that point. 
With a sliding-filament model, the variable $m$ can be controlled by the curvature, and equations (6), (13) and ( 5 ) lead to

$$
m=d M_{v} / d x=-C_{N} 2 \pi f(\lambda / 2 \pi)^{3} \kappa .
$$

In this case, $m$ varies in phase with $-\kappa$, and a simple scalar function of $\kappa$ is all that is required for the control of $m$. This function will be represented by $m(\kappa)$. No phase lag is required between $m$ and $\kappa$, because a $\frac{1}{4}$-cycle phase difference between $m$ and $M_{a}$ is introduced by equation (3). In effect, the sliding-filament model provides a frequency-independent mechanism for generating the phase lag between $M_{a}$ and $-\kappa$ required by Machin's original proposal.

Equation (II) describes sinusoidal bending waves which are propagated along the flagellum in the direction of increasing $x$; i.e. from left to right. The sign relationship between $m$ and $\kappa$ given by equation (I6) is the same as that mentioned in the previous section, which will cause propagation of a bend from left to right along the flagellum. If $m$ is proportional to $-\kappa$, the flagellum will automatically propagate bending waves with the balance between active and viscous bending moments which is required for uniform wave propagation. The sign of the function $m(\kappa)$ will determine the direction of wave propagation. If $m(\kappa)$ is a linear function of $\kappa$, and if the amplitude of the waves is kept small, sinusoidal bending waves will result. Under these conditions, equation (I6) shows that the magnitude of $m(\kappa)$ will determine the value of $f \lambda^{3}$, and therefore place one constraint on the values of the three wave parameters. Two additional factors are required to completely determine the wave parameters.

\section{Large-amplitude waves on a flagellum of infinite length}

Flagella typically generate non-sinusoidal bending waves of rather large amplitude. An analytical evaluation of the bending moments resulting from viscous forces becomes considerably more complicated. However, the viscous moments can be computed using numerical integration on a digital computer, as outlined previously (Brokaw, 1970). These computational methods have now been used to examine the distribution of $d M_{v} / d s$ along the length of a flagellum propagating several types of uniform bending wave, in which the wave parameters are constant along the length of the flagellum. To eliminate end-effects, waves having an integral number of wavelengths were examined at times corresponding to zero curvature at the ends of the flagellum. The ratio of the normal and tangential drag coefficients, $C_{N} / C_{L}$, was given the value $\mathrm{I} \cdot 8$, as in the previous computations.

Viscous bending moments were computed for three types of wave patterns: largeamplitude sinusoidal waves; sine-generated waves, in which the curvature is a sinusoidal function of length along the flagellum; and waves constructed of circular arcs and straight lines, as suggested by Brokaw \& Wright (1963). These computations showed that the distribution of viscous bending moment along a flagellum is not very sensitive to the precise form of the bending waves, even though the distributions of curvature along the length for these three types of waves are quite different. The ratio between $d M_{v} / d s$ and $\kappa$ at various points in these waves is not equal to a constant, as in equation (I6) for small-amplitude waves. This is illustrated in Fig. 2, which shows $d M_{v} / d s$ plotted against $\kappa$ at various points within sinusoidal and sine-generated waveforms. The curves in Fig. 2 indicate the form of the function $m(\kappa)$ which would be 
required to generate the corresponding waveform. These curves suggest that a linear system, which generates a value of $m$ strictly proportional to $\kappa$, would produce a waveform intermediate between a sinusoidal and a sine-generated wave. An $m(\kappa)$ function which increases more steeply with $\kappa$ than the curve shown for sine-generated waves in Fig. 2 would be required to generate bending waves which appear to be constructed of circular arcs and straight lines. Although the latter type of bending wave has been suggested to be the best representation for flagellar bending waves (Brokaw \& Wright, 1963; Brokaw, 1965), more accurate methods for measuring the curvature in photographs of flagellar wave patterns will be required for a precise definition of the function $m(\kappa)$ for a particular flagellum. Different flagella may well have different forms of $m(\kappa)$ and generate different types of bending-wave pattern.

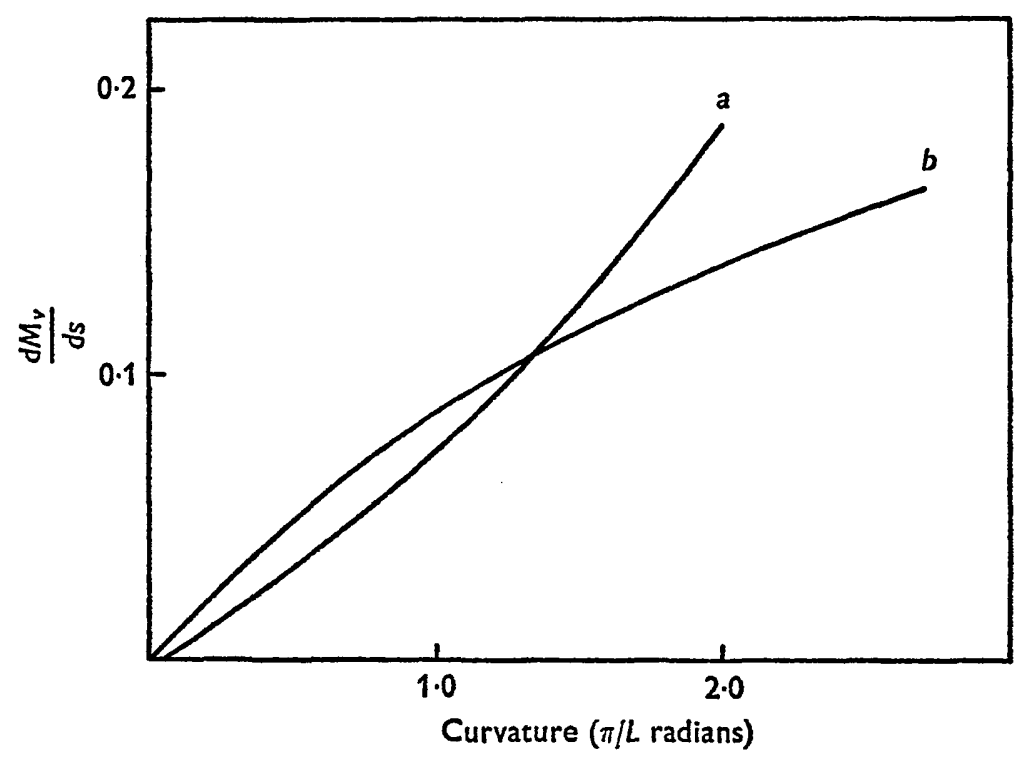

Fig. 2. Variation in $d M_{v} / d s$ with curvature, $\kappa$, within large-amplitude bending waves. Curve $a$ was obtained from computations with a sine-generated binding wave and curve $b$ from computations with a sinusoidal bending wave. The amplitudes of the waves are such that each had a total angle of 2.0 radians in each of its bends. The values given for $d M_{v} / d s$ are in dimensionless units which must be multiplied by $C_{L} f L^{2}$ to obtain values in real units.

These curves show the shape of the function $m(\kappa)$ which is required to generate the corresponding waveform according to the model proposed in this paper.

An additional effect obtained with large-amplitude waves is illustrated by Fig. 3 . Peak values of $d M_{v} / d s$ were computed for bending waves of varying amplitude. At the amplitudes normally found on flagella the peak value of $d M_{v} / d s$ does not increase in proportion to $\kappa$, as predicted by the small-amplitude equations. Consequently, the relationship between $f$ and $\lambda$ and the magnitude of $m(\kappa)$ will not be independent of amplitude, as in equation (I6) for small-amplitude movement. This conclusion can also be obtained from an earlier analytical treatment (Brokaw, 1965), but is summarized in a possibly more useful form by Fig. 3 .

\section{Uniform bending waves on flagella of finite length}

With flagella of finite length the boundary conditions which must be met at the ends of the flagellum will influence the distribution of viscous bending moment on the 
flagellum. These boundary conditions must specify the moments and forces applied to the ends of the flagellum. For an isolated flagellum which is swimming freely through a viscous medium both the external moment and the external force applied to the ends of the flagellum must be zero. This corresponds to the specification that $M_{v}=0$ and $d M_{v} / d s=0$ at $s=0$ and at $s=S$. The effects of these boundary conditions were included in the computations of viscous bending moments for largeamplitude wave movement in an earlier paper (Brokaw, 1970). The results showed that the values of $M_{v}$ at a particular phase point vary greatly over the length of the flagellum,

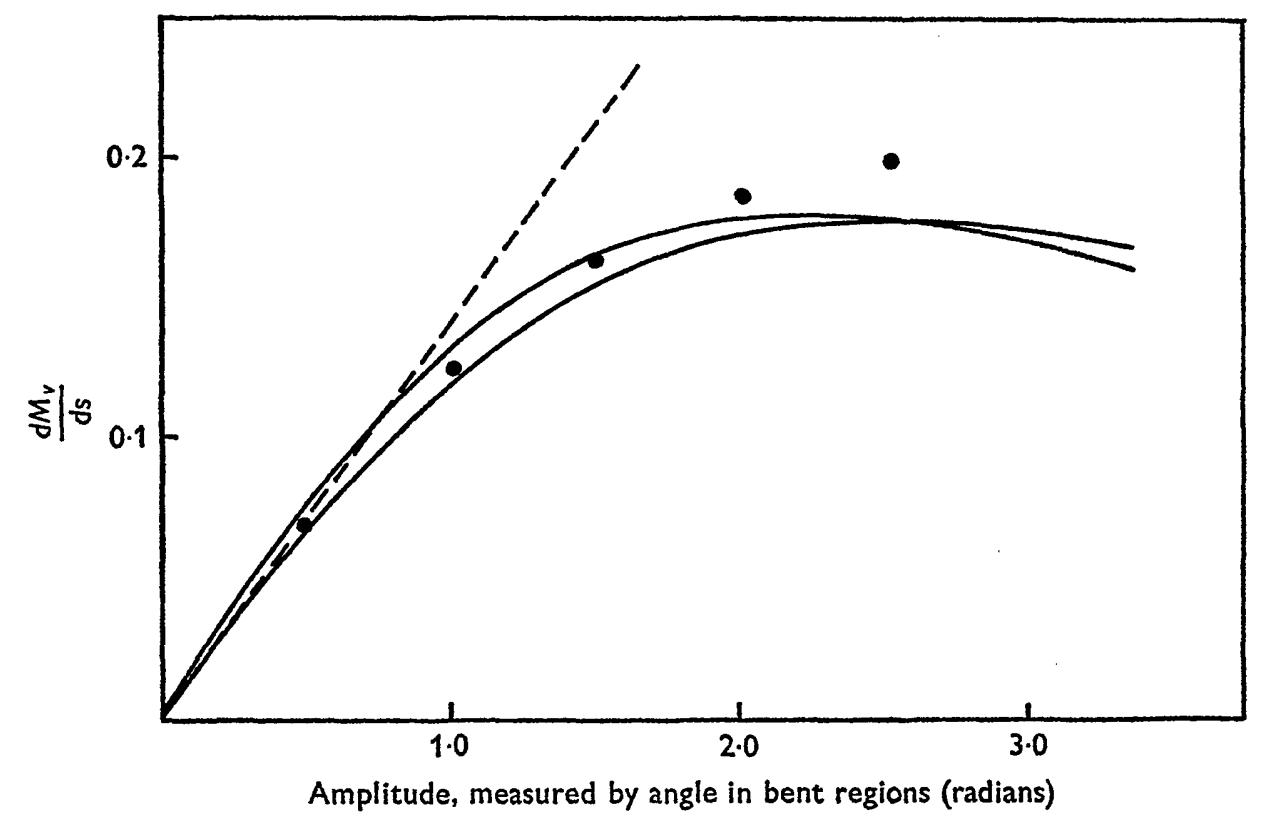

Fig. 3. Effect of wave amplitude on viscous bending moments. The wave amplitude is measured by the total angle in each bent region. The ordinate gives, in dimensionless units (see Fig. 2), the peak value of $d M_{v} / d s$, which occurs between the bends. The dashed line represents the result predicted by the small-amplitude equations, if they are written in terms of $L$, the wavelength measured along the flagellum, instead of in terms of $\lambda$, the wavelength measured along the $x$ axis. The points were computed using the methods of Brokaw (r.970) for a sinegenerated bending wave. The solid lines were calculated for bending waves constructed of circular arcs and straight lines, using equations from Brokaw (1965) and the conclusion that the distribution of $M_{v}$ is nearly sinusoidal. The fraction of the wavelength $L$ which is included in the bent regions is 0.6 for the upper curve and 0.8 for the lower curve. The divergence between the points and the lines at higher amplitudes reflects the fact that the distribution of $M_{v}$ for sine-generated waves diverges from a sinusoidal function at large amplitudes.

and implied that it would be difficult for a flagellum to control $M_{a}$ so as to keep $M_{a}+M_{v}=0$ over the entire length of the flagellum. This formed another serious objection to local bending models for flagellar bend propagation, such as that proposed by Brokaw (1966a), which required some mechanism to ensure a uniform velocity of bend propagation along the flagellum.

This problem is at least partially resolved by consideration of the sliding-filament model, where the value of $d M_{v} / d s$ at a particular phase point in the bending wave is the relevant condition to be matched by the active process controlled by $\kappa$. This can be seen from the curves labelled ' $B+U$ ' in Figs. 6-9 of Brokaw (1970), which show, over most of the length of the flagellum, the average value of $d M_{v} / d s$ within a bent region 
as the bent region travels along the flagellum. These ' $B+U$ ' curves show significantly less variation along the length of the flagellum than the curves labelled ' $B$ ' or ' $U$ ', which indicate the variation in $M_{v}$ at particular phase points (the ends of a bent region) as the bent region travels along the flagellum. These computations can also produce curves showing the distribution of $M_{v}$ and $d M_{v} / d s$ along the length of the flagellum at particular times in the beat cycle. Such curves show that the relationship between the phase of $d M_{v} / d s$ and the phase of $\kappa$ is not significantly altered by the boundary conditions introduced at the ends of the flagellum.

The influence of these boundary conditions can also be analysed by direct calculations for small-amplitude sinusoidal waves. In this case, equation (12) becomes

$$
d^{2} M_{v} / d x^{2}=-C_{N} 2 \pi f b i e^{i 2 \pi f t}\left(e^{-i 2 \pi x / \lambda}+C_{1}+C_{2} x\right) \text {. }
$$

In addition to the $d y / d t$ term resulting from the sinusoidal wave movement, equation ( 17 ) contains a term resulting from oscillation of the entire flagellum in the $y$ direction with an amplitude and phase determined by the complex constant $C_{1}$, and a term resulting from rotational oscillation of the entire flagellum around the origin with an amplitude and phase determined by the complex constant $C_{2}$. This is equivalent to the method used for large-amplitude movement, where the integrations were performed numerically (Brokaw 1970). After integration of equation (I7) and evaluation of the constants of integration to give zero values for $M_{v}$ and $d M_{v} / d x$ at $x=0$,

$$
\begin{aligned}
& d M_{v} / d x=C_{N} b 2 \pi f(\lambda / 2 \pi) e^{i 2 \pi(f t-x / \lambda)}\left\{\mathrm{I}+e^{i 2 \pi x / \lambda}\left[-\mathrm{I}+C_{1}(2 \pi x / \lambda)+\frac{1}{2} C_{2} x(2 \pi x / \lambda)\right]\right\}, \\
& \text { and } \\
& M_{v}=i C_{N} b 2 \pi f(\lambda / 2 \pi)^{2} e^{i 2 \pi(f t-x / \lambda)}\left\{\mathrm{I}+e^{i 2 \pi x / \lambda}\left[-\mathrm{I}+i 2 \pi x / \lambda-\frac{1}{2} C_{1}(2 \pi x / \lambda)^{2}\right.\right. \\
& \left.\left.-C_{2} x(2 \pi x / \lambda)^{2} / 6\right]\right\} .
\end{aligned}
$$

$C_{1}$ and $C_{2}$ are then evaluated to give zero values for $M_{v}$ and $d M_{v} / d x$ at the distal end of the flagellum, where $x=S$ :

and

$$
C_{1}=6(\lambda / 2 \pi S)^{2}\left(e^{-i 2 \pi S / \lambda}-\mathrm{I}\right)+i(\lambda / \pi S)\left(e^{-2 \pi S / \lambda}+2\right)
$$

$$
C_{2}=-\mathrm{I} 2(\lambda / 2 \pi S)^{2}\left(e^{-i 2 \pi S / \lambda}-\mathrm{I}\right) / S-i 6(\lambda / 2 \pi S)\left(e^{-i 2 \pi S / \lambda}+\mathrm{I}\right) / S .
$$

The first terms in equations (I8) and (I9) give the results obtained in equations (I3) and (14). With these expressions it is possible to examine, for instance, the values of $M_{v}$ corresponding to points on the wave where $\kappa=0$, and the values of $d M_{v} / d x$ at the points on the wave corresponding to the peak values of $\kappa$, as these phase points travel along the length of the flagellum. Curves showing the variation in these values along the length of the flagellum are shown in Fig. 4, for various values of $S / \lambda$, the ratio between the length of the flagellum and the wavelength of the bending waves. The influence of the end-effects on small-amplitude sinusoidal movement is seen to introduce most of the variation in $M_{v}$ and $d M_{v} / d s$ along the length of the flagellum which was obtained with the earlier computations for large amplitude waves (Brokaw, I970).

In uniform bending waves the value of $\kappa$ at a particular phase point remains constant as this phase point travels along the flagellum. The curves in Fig. 4 indicate that the variation in the value of $d M_{v} / d x$ at a phase point travelling along the flagellum is much less than the variation in $M_{v}$. A control mechanism relating $m$ and $\kappa$ is therefore much more reasonable than one relating $M_{a}$ and $\kappa$, as the resulting waves will be much less 
strongly affected by the boundary conditions at the ends of the flagellum. However, there are still variations in the value of $m$ required to match $d M_{v} / d x$ for the propagation of uniform bending waves which cannot be completely overlooked. With uniform waves it is impossible for $m(\kappa)$ to be constant, to match $m(\kappa)$ to $d M_{v} / d x$, and also to satisfy the boundary condition that $d M_{v} / d x=0$. At the distal end of the flagellum this problem might be partially resolved by having a short length of inactive flagellum, in which $m=0$, at the distal end. In fact, the flagella of spermatozoa such as seaurchin spermatozoa have a thin 'terminal piece' at the distal end, which has a simplified ultrastructure, and might well be a passive appendage. Removal of the normal distal end of the flagellum interferes with the ability of the flagellum to propagate uniform

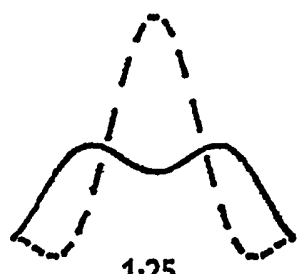

$1 \cdot 25$

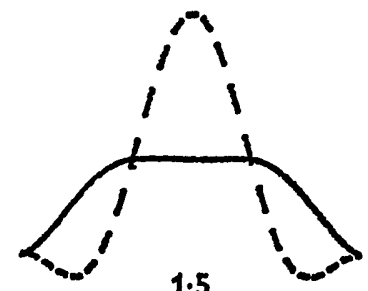

1.5

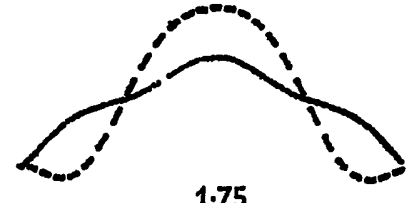

1.75
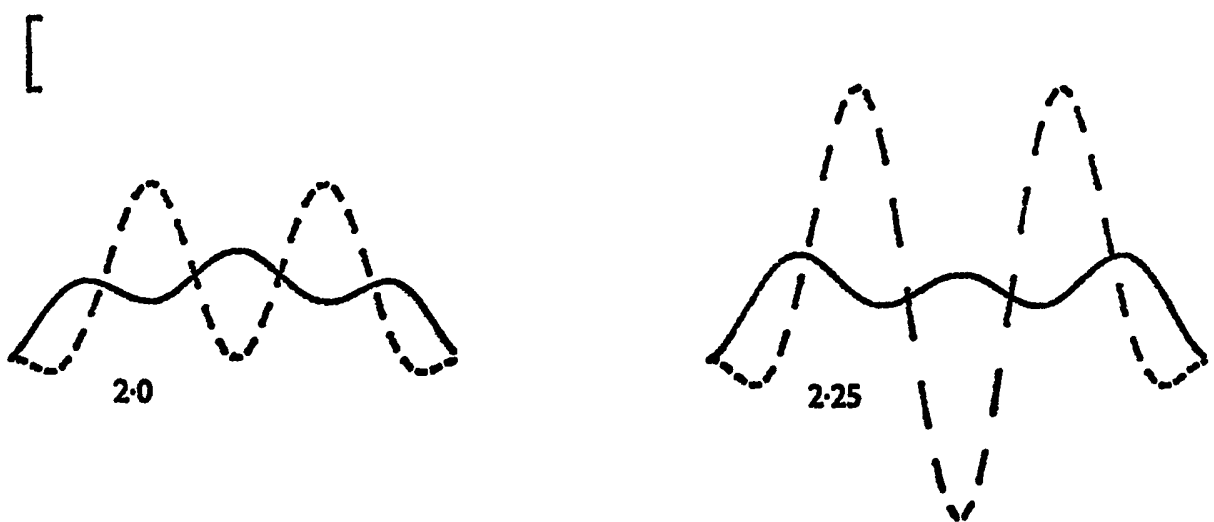

Fig. 4. Variation in the peak values of $d M_{v} / d x$ (solid curves) and $M_{v}$ (dashed curves) along the length of flagella of finite length propagating small-amplitude sinusoidal bending waves. The ratio, $S / \lambda$, between the length of the flagellum and the wavelength of the bending waves is indicated under each curve. The ordinate scale marker indicates the values given for waves on a flagellum of infinite length by equations (13) and (14).

bending waves all the way to the distal end of the flagellum (Brokaw, 1965). This observation was originally interpreted as evidence for a sliding filament type of model in which interaction between the filaments was restricted to the straight regions between bends (Brokaw, 1968). A better interpretation is suggested by the above discussion, but a more detailed examination of the bending patterns of flagella after removal of the distal end would be valuable.

The inverse problem of calculating the degree of non-uniformity in the propagation of flagellar bending waves which would result from the effects of the boundary conditions at the ends of the flagellum on the distribution of $d M_{v} / d s$ is a much more difficult mathematical problem. This problem requires further study before the proposed mechanism for the control of flagellar wave propagation can be thoroughly evaluated. 


\section{Asymmetrical and non-uniform bending patterns}

Bending waves on real flagella are often asymmetrical and are never completely uniform, because the bends start with smaller dimensions near the base of the flagellum and enlarge as they accelerate to the propagation velocity which is maintained over most of the flagellum (Gray, r955; Brokaw, 1965, 1970). This non-uniformity, and a small degree of asymmetry, were included in the computations of viscous bending moments published previously (Brokaw, 1970); further computations have since been carried out for flagella propagating bending waves with more extreme degrees of asymmetry.

The major conclusion indicated by computations for asymmetrical bending waves is that in these waves $d M_{v} / d s$ is not a symmetrical function of $\kappa$. In the bent regions having very low values of $\kappa, d M_{v} / d s$ has values which are nearly as great as in the bent regions of higher curvature. If asymmetrical bending waves are to be generated by the control mechanism suggested in this paper, the transfer function between $m$ and $\kappa$ must be asymmetrical.

The similarity between the asymmetrical bending patterns which can be generated by flagella and bending patterns generated by cilia has been pointed out (Sleigh, I962), and some information is available about the distribution of viscous bending moments in cilia (Rikmenspoel \& Sleigh, I970). The 'straight region' which occupies most of the length of a cilium during its effective stroke may be equivalent to a bent region of very low curvature in a flagellum propagating asymmetrical bending waves, rather than the straight regions between bends in a flagellum propagating symmetrical waves. In this case a control mechanism similar to that suggested here for flagella might also be able to generate the bending patterns found on cilia. Analysis of this possibility requires further examination of solutions to equation (10) with boundary conditions appropriate for cilia and an asymmetrical relationship between $m$ and $\kappa$.

The results published previously (Brokaw, 1970), and other computations, also show that in the region near the base of the flagellum where bends are initiated there is no obvious relationship between the bending of the flagellum and the distribution of $d M_{v} / d s$ or $M_{v}$. Although the behaviour of the flagellum in this region is not independent of the viscous moments on the flagellum, since the frequency of the whole flagellum is sensitive to the viscosity of the medium, other factors must be involved in controlling bend initiation. This problem will not be dealt with in the present paper.

\section{THE INFLUENCE OF ELASTIC RESISTANCES WITHIN THE FLAGELLUM}

In the local-bending model considered by Machin (1958), the elasticity of the flagellum is introduced by using equation (7) and a single elastic constant, $\alpha$, for the bending resistance of the flagellum. The presence of an elastic bending resistance increases the phase lag required between $-\kappa$ and $M_{a}$ from $\frac{1}{4}$ cycle to a maximum of $\frac{1}{2}$ cycle, depending on the magnitude of $\alpha$. The control mechanism must generate a phase lag restricted to this range, but the phase lag no longer has to be completely independent of frequency. However, since flagella can operate over a wide range of frequencies, it will still be difficult to generate the necessary phase lag by a simple time delay process. 
With the sliding-filament model, small-amplitude sinusoidal movement will be a solution to equation (I0) if the transfer function between $\kappa$ and $m$ has the form

$$
m=(A-i B) \kappa .
$$

$A$ and $B$ are related to the wave parameters by

as in equation (16), and

$$
A=-C_{N} 2 \pi f(\lambda / 2 \pi)^{3},
$$

$$
B=-2 \alpha_{0}(2 \pi / \lambda)-r^{2} \alpha_{2}(\lambda / 2 \pi) .
$$

This solution requires that the transfer function introduce a phase lag between $-\kappa$ and $m$, corresponding to a phase angle, $\phi$, given by

$$
\tan \phi=B / A \text {. }
$$

This phase lag will be small if the values of $\alpha_{0}$ and $\alpha_{2}$ are small. Unless $\alpha_{0}$ and $\alpha_{2}$ are very large, there is no restriction on the variation of this phase lag with frequency, so that it can be generated by some simple time-delay processes. Since the transfer function now introduces two variables, corresponding to $\phi$ and the magnitude of $m$, it may determine both $f$ and $\lambda$ for the resulting small-amplitude sinusoidal movement.

With large-amplitude bending waves a simple exact solution to equation (Io) requires that if the magnitude of $m$ is not a linear function of $\kappa$, there must be a similar non-linearity in the behaviour of the elastic elements of the flagellum as $\kappa$ increases. Similarly, if asymmetrical bending is generated by an asymmetrical transfer function between $m$ and $\kappa$, an exact solution for uniform wave movement requires that the asymmetry in $m$ is matched by similar asymmetry in the behaviour of the elastic elements of the flagellum. This exact matching appears unlikely. It seems more probable that if $\alpha_{0}$ and $\alpha_{2}$ are sufficiently small, bending-wave propagation will still be possible even if the elastic properties of the flagellum do not satisfy the conditions for an exact solution for uniform waves, and there will be small non-uniformities in the parameters of the propagated bending waves. However, this has not yet been demonstrated.

To solve equation (Io) for a flagellum of finite length, not only must the boundary conditions for $M_{v}$ and $d M_{v} / d s$ at the ends of the flagellum be satisfied, but, in addition, the solution will depend on the boundary conditions specified for the shear system. A reasonable set of boundary conditions for shear might be $u=0$ at the base of the flagellum and, at the distal end of the flagellum, the condition that the sum of $M_{a}$ and the elastic moment resulting from shear resistance equals zero. It seems possible that these boundary conditions might be relevant to understanding the non-uniform bending behaviour near the base of the flagellum, where bends are initiated, but this problem also remains for future study.

\section{DISCUSSION}

The preceding discussion suggests that, if the active process responsible for the bending of cilia and flagella is a 'sliding filament' process similar to that which has been suggested for muscle, a very simple control mechanism can explain the regular propagation of bends along flagella. This mechanism requires only that the local magnitude of the active moment per unit length generated by the active 'sliding filament' process is controlled locally by the curvature of the flagellum. This mechanism 
for the control of bend propagation combines elements of two propagation mechanisms suggested previously for flagella (Machin, 1958; Brokaw, 1966a). The modifications introduced in adapting these two mechanisms to flagella in which bending is generated by a sliding-filament process result in a resolution of the main difficulties which appeared when these propagation mechanisms were developed in terms of a 'localbending' model for flagella.

The behaviour of the proposed model for flagella is summarized by a differential equation, equation (10). Only some properties of solutions to this equation have been discussed, but the discussion suggests that solutions to this equation exist which will match the behaviour of real flagella. Further study of methods for finding solutions to equation (I0) which will satisfy realistic boundary conditions at the ends of the flagellum and incorporate specific forms of the relationship between curvature and active moment is needed.

The proposed mechanism for bend propagation implies that once a bend is established on a flagellum it will be propagated to the distal end without requiring any controlling information from the base of the flagellum. The parameters of the propagated bend will be determined primarily by local properties of the flagellum and by the viscous resistance to its movement. This mechanism is therefore largely consistent with the results of Goldstein (1969), who found that bends would continue to be propagated after sudden breakage of a sperm flagellum by a laser microbeam. Until bend initiation is studied in detail, it is difficult to relate the proposed mechanism to the observations that after breakage of a sea-urchin sperm flagellum with a laser microbeam no new bends are initiated distal to the point of breakage (Goldstein, 1969), while the flagellum of a protozoan, Crithidia oncopelti can initiate new bends after similar experimental removal (Goldstein, Holwill \& Silvester, 1970). However, in the sliding-filament model for flagella, bending of the flagellum depends not only on the active process which generates longitudinal shear, but also on the presence of longitudinal shear resistance, which is essential to convert active sliding into bending. With a sliding-filament model the absence of bend initiation in a flagellar fragment might be attributed to the absence of the normal amount of shear resistance near the base of the flagellum. Other flagella might have sufficient shear resistance distributed along the length of the flagellum to allow bend initiation to occur in the absence of the normal basal end of the flagellum.

The propagation mechanism outlined here does not in itself predict how the parameters of flagellar movement, such as the frequency and the wavelength, will behave under experimental conditions such as variations in viscosity, nor does it allow values for the elastic constants of the flagellum to be calculated from measurements of the parameters of movement. However, it does suggest a basis for relating the movement parameters to parameters for the active process, so that particular kinetic models for the active process can be examined in relation to the experimental data on movement parameters. The analysis of models for the kinetics of the active process is a logical extension of the present study, but there are also other areas which require further study. Perhaps the most important is the analysis of bend initiation, in order to see whether this process also forms part of the solution to equation (ro) with appropriate specification of the relationship between curvature and the active process, and of the boundary conditions. In addition, there is a need for further study of the application of the proposed control mechanism to the generation of ciliary beating patterns and 


\section{Sliding-filament model for flagella}

to the generation of three-dimensional beating patterns by cilia and flagella. An understanding of the propagation of three-dimensional bending patterns will undoubtedly require that the specification of the sliding-filament model be extended to match the unique pattern of filaments actually found in flagella. This extension is also a prerequisite for an understanding in molecular terms of the proposed relationship between the curvature of the flagellum and the generation of active sliding between the filaments.

\section{SUMMARY}

I. If active sliding occurs between flagellar filaments, as suggested for the sliding filament mechanism for muscular contraction, it will generate positive bending in one direction along the flagellum and negative bending in the opposite direction. Bends will therefore be propagated automatically if the sliding process is activated by bending.

2. The active bending moment required to match the viscous bending moments resulting from the uniform propagation of bending waves can be generated by a simple relationship between the curvature of the flagellum at any point and the magnitude of the moment per unit length generated by the active sliding process at that point.

3. This propagation mechanism incorporates features of the two mechanisms proposed previously for flagellar-bend propagation. The major difficulties which arise when these propagation mechanisms are developed in terms of a 'local-bending' model for flagellar bending are avoided when a 'sliding filament' model is used.

4. The elastic constants of the flagellum have a minor role in bend propagation, and without additional assumptions their magnitudes cannot be obtained from measurements of the parameters of flagellar bending-wave propagation.

I am grateful to the John Simon Guggenheim Foundation for financial support which has made this work possible, and to Dr K. E. Machin for extensive and vital discussions of the manuscript.

\section{REFERENCES}

Brokaw, C. J. (1962). Studies on isolated flagella. In D. W. Bishop (ed.), Spermatozoan Motility, pp. 269-78. A.A.A.S., Washington, D.C.

Brokaw, C. J. (1965). Non-sinusoidal bending waves of sperm flagella. $\mathcal{F}$. exp. Biol. 43, 155-69.

Brokaw, C. J. (1966a). Bend propagation along flagella. Nature, Lond. 209, 16I-3.

Brokaw, C. J. (1966 b). Effects of increased viscosity on the movements of some invertebrate spermatozoa. F. exp. Biol. 45, I13-9.

Brokaw, C. J. (1968). Mechanisms of sperm movement. Symp. Soc. exp. Biol. 22, 101-16.

Brokaw, C. J. (1970). Bending moments in free-swimming flagella. F. exp. Biol. 53, 445-64.

Brokaw, C. J. \& Wright, L. (1963). Bending waves of the posterior flagellum of Ceratium. Science, N.Y. 142, I $169-70$.

Gray, J. (1955). The movement of sea-urchin spermatozoa. F. exp. Biol. 32, 775-801.

GoldSTEIN, S. F. (1969). Irradiation of sperm tails by laser microbeam. F. exp. Biol. 5I, 43I-4I.

GoldsteIn, S. F., Holwill, M. E. J. \& Silvester, N. R. (I970). The effects of laser microbeam irradiation on the flagellum of Crithidia (Strigomonas) oncopelti. F. exp. Biol. 53, 401-9.

HorRidge, G. A. (1965). Macrocilia with numerous shafts from the lips of the ctenophore Beroë. Proc. R. Soc. B I62; $35 \mathrm{I}-64$.

LUBliner, J. \& BLUM, J. J. (1971). Model for bend propagation in flagella. F. theor. Biol. 31, 1-24.

MACHIN, K. E. (1958). Wave propagation along flagella. F. exp. Biol. 35, 796-806.

Machin, K. E. (1963). The control and synchronization of flagellar movement. Proc. R. Soc. B 158, 88I04.

MAcHIN, K. E. (1964). Feedback theory and its application to biological systems. Symp. Soc. exp. Biol. I8. $42 I-46$.

RikmenspoEL, R. (1965). The tail movement of bull spermatozoa. Observations and model calculations. Biophys. F. 5, 365-92. 
RIKMENSPOEL, R. (1966). Elastic properties of the sea urchin sperm flagellum. Biophys. F. 6, 47I-80. RiKMENSPOEL, R. \& SLEIGH, M. A. (I970). Bending moments and elastic constants in cilia. F. theor. Biol. 28, 8I-100.

SATIR, P. (1965). Studies on cilia. II. Examination of the distal region of the ciliary shaft and the role of the filaments in motility. F. Cell Biol. 26, 805-834.

SatiR, P. (1967). Morphological aspects of ciliary motility. F. gen. Physiol. 5o, (6-2; suppl.), 241-258.

SATIR, P. (r968). Studies on cilia. III. Further studies of the cilium tip and a 'sliding filament' model of ciliary motility. F. Cell Biol. 39, 77-94.

Sleigh, M. A. (1962). The Biology of Cilia and Flagella. Pergamon Press.

SLeIGH, M. A. (1968). Patterns of ciliary beating. Symp. Soc. exp. Biol. 22, I31-50. 